\title{
A design of solid state power controller based on FPGA
}

\author{
Liu Jianying, Gao Kai, Yang zhangang,Yu Haiting,Yan chaoqi \\ Civil Aviation University of China, Tianjin 300300, China \\ 15222126212@163.com
}

Keywords: aviation equipment; solid state power controller; inverse time over-current protection; reaction speed; protection algorithm; trip.

\begin{abstract}
With the increasing power demand of aviation equipment, the requirement of the protection of electrical load on the plane is more and more stringent. Once the over-current happened, it would be required that the system must quickly cut off the fault circuit. A solid state power controller based on FPGA had been designed, and the principle of the inverse time over-current protection has been introduced, the protection algorithm has been analyzed and studied in detail. Finally, the experimental test had been done on the prototype in the laboratory, the actual test result shows that the solid state power controller response speed is very fast, which can quickly trip to protect the circuit if there is an over-current. The measured curve is conformed with the theoretical assumption. Therefore, it has a significant practical value.
\end{abstract}

\section{Introduction}

With the development of modern aviation technology, the aircraft electrical system is more and more complex, and the performance is more perfect than before. Therefore, the requirements for the modern aircraft are more stringent than the traditional aircraft. As shown in Figure 1, the advanced aircraft power distribution system mainly consists of the power system processor (PSP), the electrical load management center (ELMC), and the solid state power controller (SSPC). In the process of flight, the power system processor is integrated management and processing of power distribution system. Load management center is mainly to achieve secondary power distribution, protect and monitor the load. Solid state power controller has the function of protecting the circuit overload and short circuit. Once the over-current happened, the system should be able to react quickly and cut off the fault circuit in order to avoid the damage of the whole system. Therefore, the application of SSPC technology is very strict, one of the more mature technology is the inverse time over-current protection.

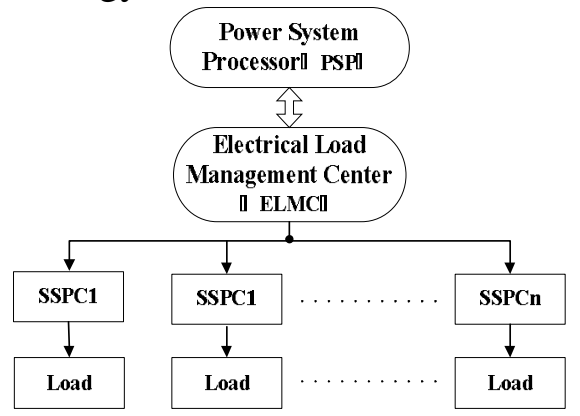

Fig.1 Aircraft advanced distribution system structure diagram 


\section{The composition and basic principle of the solid state power controller}

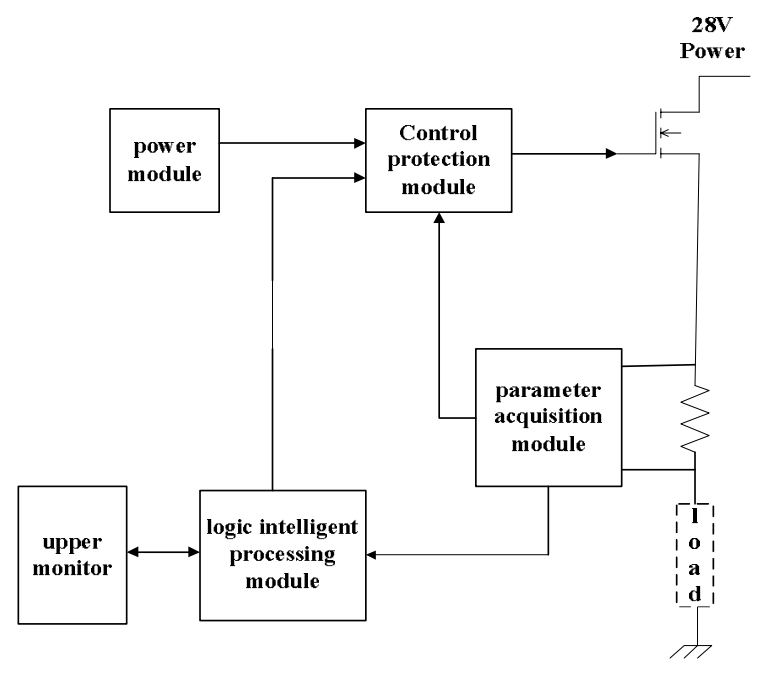

Fig.2 Principle diagram of solid state power controller (SSPC)

As shown in figure 2, the solid state power controller is composed of four parts: power module, control protection module, logic intelligent processing module and parameter acquisition module.

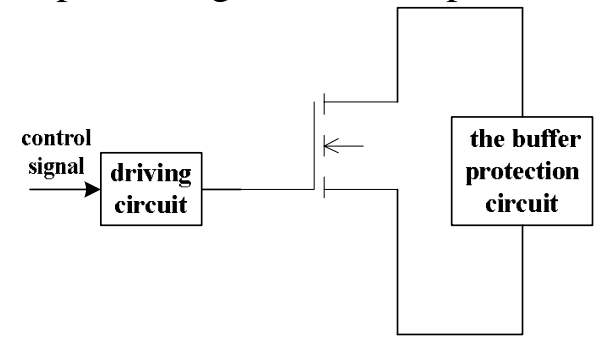

Fig.3 Component of control protection module

Control protection module. Control protection module mainly realizes the buffer protection of SSPC and driving control the MOSFET tube etc. As shown in Figure 3, it mainly consists of the driving circuit and the buffer protection circuit. The driving circuit can drive the MOS tube's break-over and turn off. The main function of the buffer protection circuit is to prevent the transient overvoltage, over current and the switching loss of the device in the MOS tube.

Logic intelligent processing module. Logic intelligent processing module can realize the communication between the host computer and SSPC, upload SSPC status information, and receive the upper monitor's control command to control the break-over or shut-off of the MOSFET, realization of inverse time algorithm for load over current protection, collect and store the fault information of the load etc.

The CYCLONE IV EP4CE22F17 chip of the ALTERA corporation has been chosen to as the logic processing module.

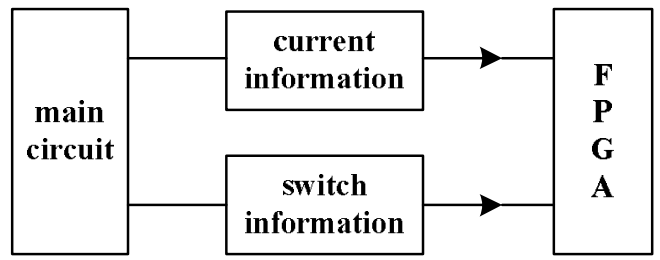

Fig.4 Parameter acquisition principle

Parameter acquisition module. As shown in figure 4, the parameter acquisition module collects the status information and uploads the collected signal after filter amplifier and isolation, combined with the state output, then the state of SSPC can be decided.

Power module. Power module provides the internal power for the SSPC. The power for the circuit internal chip can be obtained after the input DC voltage is converted by the power module. 


\section{Inverse time over-current protection principle}

Each load and MOSFET tube has the maximum limit current value. Therefore, when the short-circuit flow occurs in the circuit, the current may instantly reach hundreds of ampere, which can cause the damage of the electronic components. Thus the over-current protection is essential in the circuit. In order to prevent the components from damaging, the protection measure taken when the circuit current is going to achieve the rated limit of the components is called over-current protection. When the over-current fault occurs, the circuit should be able to quickly response and the power tube should be cut off immediately. In many practical applications, $I^{2} t$ protection curve is the priority choice. The component's in a circuit is equal to the product of the current's square and the resistance of the component. The temperature of the component is determined by the product of its power and the time. Therefore, in the circuit, the temperature is proportional to the square of the current. The inverse time protection is based on the above concept.

Inverse time protection is a secondary protection method. The greater the short circuit current is, the faster the protection action will be when the over-current fault occurs in the circuit. The $\mathrm{I}^{2} \mathrm{t}$ signal shows the accumulation of the temperature in the circuit. The temperature that some components can endure is a constant value, if the temperature is higher than this limit value, the components will be damaged .Therefore, when SSPC is designed, the inverse time over-current protection should be designed to enable the system to cut off the MOSFFT rapidly when the circuit current value is reaching the limit value.

\section{Derivation of inverse time over-current algorithm}

At present, the unified mathematical model of the inverse time protection tripping time can be presented as

$$
\mathrm{T}=\frac{\mathrm{K}}{\left(\frac{i}{I_{p}}\right)^{r}-1}
$$

where, ' $i$ ' is the fault current; ' $I_{p}$ ' is the protection starting current; ' $r$ ' is a constant whose value is usually between 0 to 2 ; and ' $K$ ' is a constant.

According to the requirements of the British Standard Specification (BS5142) and the International Electrical Commission (IEC255), three standard inverse time characteristic equations are generally used. which are shown as follows.

The general inverse time characteristics

$$
\mathrm{t}=\frac{0.14 \mathrm{t}_{p}}{\left(\frac{i}{I_{p}}\right)^{0.02}-1}
$$

very inverse time characteristics

$$
\mathrm{t}=\frac{13.5 \mathrm{t}_{p}}{\left(\frac{i}{i_{p}}\right)-1}
$$

and super inverse time characteristics 


$$
\mathrm{t}=\frac{80 \mathrm{t}_{p}}{\left(\frac{i}{i_{p}}\right)^{2}-1}
$$

where ,' $\mathrm{t}$ ' is trip time interval; ' $t_{p}$ ' is a time constant; ' $i_{p}$ ' is the current value of inverse time protection during MOSFET working, that is, the rated current value of the MOSFET; ' $i$ ' is the current value when failure occurs; ' $i / i_{p}$ ' is the overload ratio. Super inverse time curves are used in experiments. The characteristic curve of inverse time protection action can be obtained by using MATLAB. On condition that $\mathrm{k}=1$, that is $t_{p}=1 / 80$, the characteristic curve of inverse time protection action can be shown in figure 5 .

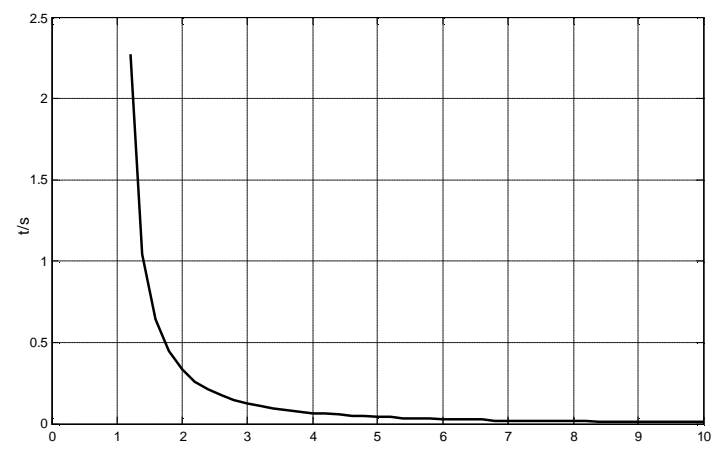

Fig.5 Inverse time curve of MATLAB

It can be transformed into the integral form

$$
\mathrm{k}=\int_{0}^{t}\left[\left(\frac{i(t)}{i_{p}}\right)^{r}-1\right] d t
$$

where the right part of the integral equation shows the accumulation of thermal effect of over-current over time. When the integration is larger than $k$, inverse time over-current protection will work.

For the FPGA can only send and receive digital signals, the formula (5) should be discretized, as shown in formula (6)

$$
\sum_{n=0}^{M}\left[\left(\frac{i(t)}{i_{p}}\right)^{r}-1\right]=\frac{k}{\Delta T}
$$

where, ' $E(n)$ ' is the current value of the nth sampling; ' $\tilde{z}_{p}$ ' is the rated current of the load; 'M' is the times of sum; and ' $\Delta T$ ' is the sample interval.

\section{Software design of inverse time over-current protection}

Solid state power controller can be applied to some basic common loads. Sometimes when the current is too large, some components would be immediately damaged because of limited capacity. In order to prevent the occurrence of this situation, when designing the inverse time over-current protection, the action time has been adjusted as follows: when the current is within the rated range, that is, the ${ }^{t} / t_{p}$ is not greater than 1, SSPC should be able to work properly-without the occurrence of tripping action. When $t_{i}$ is greater than 1 and the current exceeds the rated current $\left(t_{i}>8\right)$, the solid state power controller will trip immediately. If $i / i_{p}$ is not greater than 8 , then the accumulative value would be calculated and compared with the critical value $\frac{k}{\Delta T}$. If the accumulated value is less than the critical 
value, then return and repeat. Or else, the controller will trip immediately to disconnect the MOSFET tube. The flow chart of the inverse time over-current protection algorithm implementation is shown in figure 6 .

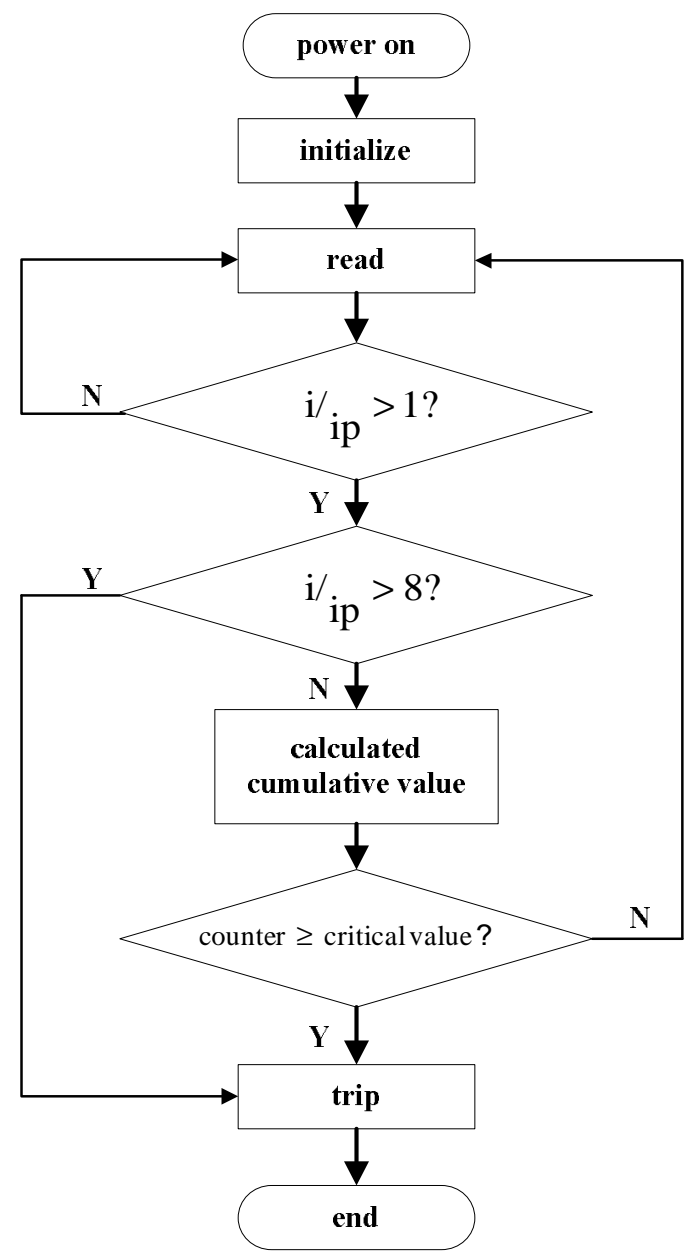

Fig.6 Inverse time over-current protection algorithm implementation flow chart

\section{Experimental Test}

In laboratory test, when the rated current is $1 \mathrm{~A}$ and $\Delta \mathrm{T}=1 \mathrm{~ms}$, the current limit value is 7 time than rated current. With the differentvalue of ${ }^{i} / i_{p}$, the corresponding shut-off time of the MOSFET tube time are as shown in Table 1.

Table 1 Inverse time protection time results

\begin{tabular}{ccc}
\hline $\begin{array}{c}\text { electric } \\
\text { current } \\
\text { ( A ) }\end{array}$ & $\begin{array}{c}\text { overload } \\
\text { multiples } \\
i_{i}\end{array}$ & $\begin{array}{c}\text { turn-off } \\
\text { time } \\
(\mathrm{S})\end{array}$ \\
\hline 1.5 & $150 \%$ & 1.05 \\
2.0 & $200 \%$ & 0.41 \\
3.0 & $300 \%$ & 0.15 \\
4.0 & $400 \%$ & 0.074 \\
5.0 & $500 \%$ & 0.043 \\
6.0 & $600 \%$ & 0.022 \\
7.0 & $700 \%$ & 0.015 \\
\hline
\end{tabular}

The test data is converted to graphic through Matlab software, as shown in figure 7. 


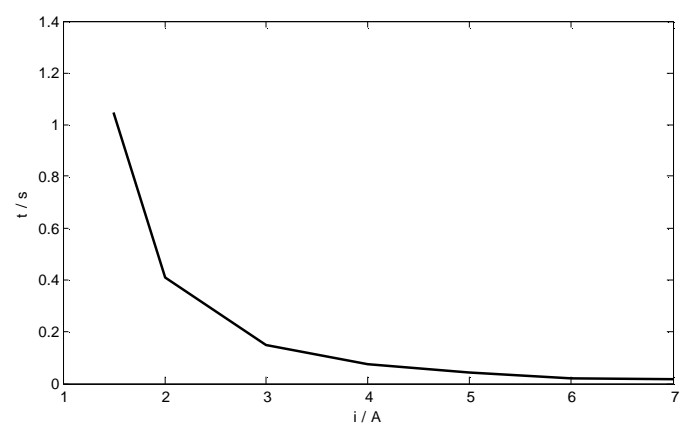

Fig.7 Inverse time experimental curve

As shown in table 1 and figure 7, the action time decreases with the increase of the load current in the test, which is consistent with concept of the inverse time limit. It can be seen from the experimental results that the whole experiment is succeeded and design meets the requirement of inverse time.

\section{Conclusions}

With the development of the modern aeronautical technology, the avionics equipment has been greatly increased on the plane. Once there is fault in the circuit, the system should be able to respond quickly so as not to affect the operation of the whole system. Using the MOSFET tube as the power switch, SSPC has the advantages of non-contact, no arc, no noise, fast response, light weight and easy to control. SSPC can be perfectly applied to the electrical equipment. A solid state power controller based on FPGA in which the inverse time protection is applied has been designed in the experiment. Finally, the experiment has been carried out on the prototype. The experimental result is consistent with theoretical assumption. Therefore, SSPC has very good practical value in practical application.

\section{Acknowledgements}

This work was financially supported by the National Natural Science Foundation of China(51407185).

\section{References}

[1] Y.V. Panov and F.C. Lee. Modeling and Stability Analysis of a DC Power System with Solid State Power Controllers[C].IEEE. San Jose, CA .March 1996.Page(s):685 - 691 vol.2

[2] M.M.R. Ahmed and P.A. Mawby. Design Specification of a 270 V 100 A Solid-State Power Controller Suitable for Aerospace Applications[C].IEEE. Barcelona. September 2009. Page(s):1 8

[3] Rosero, J. A.; Ortega, J. A.; Aldabas, E. and Romeral, L, "Moving Towards a More Electric Aircraft". Aerospace and Electronic Systems Magazine, IEEE Volume 22, Issue 3, March 2007.

[4] J. S. Cloyd, "Status of United States Air Force's More Electric Aircraft Initiative", IEEE Aerospace and Electronic Systems Magazine, vol. 13, issue 4, pp. 17 - 22, 1998

[5] M. A. Maldonado, N. M. Shah, K. J. Cleek, P. S. Walia, G. J. Korba,"Power Management and Distribution System for a More-Electric Aircraft (MADMEL) - program status”, in Proc. IECEC, vol. 1, pp. $274-279,1997$

[6] D. C. Hopkins, Y. B. Guo, H. E. Dwyer, J. D. Scofield, "Development of a SiC SSPC Module with Advanced High Temperature Packaging," High Temperature Electronics (HiTEC),May 11-13, 2010, Albuquerque, NM. 